\title{
Genetic Analysis of Faropenem-resistant Enterococcus faecalis in Urinary Isolates
}

\author{
Noriyuki Hiraga, Tetsuro Muratani, Seiji Naito, Tetsuro Matsumoto
}

Received: January 18, 2008 / Accepted: April 4, 2008

(C) Japan Antibiotics Research Association

\begin{abstract}
We isolated faropenem-resistant Enterococcus faecalis in urine specimens and studied the mechanisms of resistance to faropenem in these isolates. Three mechanisms of penicillin resistance have been reported in E. faecalis; (1) beta-lactamase production, (2) overproduction of penicillin-binding protein (PBP) 4 or PBP5, and (3) decreasing affinities of penicillins for PBP4 by the occurrence of point mutations of the penicillinbinding domain. None of the E. faecalis isolates examined produced beta-lactamase or overproduced any PBPs, but the affinities of faropenem for PBP4 were decreased in faropenem-insensitive and -resistant strains. We found single amino acid substitutions at positions 475,520 or 605 in PBP4 in the insensitive strains and two amino acid substitutions at positions 520 and 605 in PBP4 in the resistant strains by sequencing the entire $p b p 4$ gene from each isolate. We conclude that development of resistance to faropenem in E. faecalis is due to decreasing affinities for PBP4 that are the result of the occurrence of one or two point mutations.
\end{abstract}

Keywords Enterococcus faecalis, faropenem, penicillinbinding proteins, urinary tract infections

\section{Introduction}

Faropenem is a penem antibiotic, a discrete class of betalactams commonly used in the treatment of a wide range of infections including urinary tract infections (UTIs).

N. Hiraga (Corresponding author), S. Naito: Department of Urology, Graduate School of Medical Sciences, Kyushu University, 3-1-1 Maidashi, Higashi-ku, Fukuoka 812-8582, Japan, E-mail: nhiraga@med.uoeh-u.ac.jp
Faropenem is stable to beta-lactamases and has potent antimicrobial activity against Gram-positive pathogens including enterococci and Gram-negative pathogens with the exception of Pseudomonas aeruginosa [1, 2]. Accordingly, faropenem shows potent activity against most of the causative isolates of UTIs, especially against $E$. faecalis which is intrinsically resistant to cephems [3, 4]. Faropenem is often used for oral therapy in patients with UTIs in Japan [5].

Enterococci including E. faecalis and E. faecium are natural inhabitants of the gastrointestinal tract of humans. They are a common cause of UTIs and wound infections. Enterococci are the causative isolates in 3.2 to $16 \%$ of the UTIs in adults and 4.2 to $16.8 \%$ of those in children [6 9]. They can disseminate from the gastrointestinal tract to cause cholangitis, peritonitis and intra-abdominal abscess. Bacteremia and endocarditis are other wellrecognized clinical manifestations $[10,11]$. For these reasons, among causative pathogens of UTIs, enterococci are one of the most important pathogens. Among UTIcausing enterococci, multi-drug resistant $E$. faecalis such as vancomycin-resistant strains (VRE) have been reported increasingly in many countries, and in Japan the isolation frequency is similar and has increased alarmingly $[12,13]$. Most isolates of E. faecium are already resistant to penicillins, cephems and penems $[1,2]$, but $E$. faecalis is susceptible to and can be treated with penicillins including imipenem and faropenem $[1,2,14]$.

Penicillin-resistant $E$. faecalis strains have been isolated infrequently, so there have been only a few reports about

N. Hiraga, T. Muratani, T. Matsumoto: Department of Urology, School of Medicine, University of Occupational and Environmental Health, Kitakyushu, Japan 
penicillin-resistant E. faecalis. Three mechanisms of resistance to penicillins in E. faecalis have been reported: (1) the production of beta-lactamase, which hydrolyse penicillins enzymatically [15 19]; (2) overproduction of penicillin-binding protein (PBP) 4 and PBP5 [20 22]; and (3) decreasing affinities of PBP4 for penicillins by the occurrence of point mutations in the penicillin-recognition domain [23].

E. faecalis is usually susceptible to faropenem, and we have found few reports of resistance to faropenem in $E$. faecalis; however we have isolated faropenem-resistant $E$. faecalis from the urine of patients with UTIs. In the present study, we examined the mechanisms of resistance to faropenem in these isolates.

\section{Materials and Methods}

\section{Strains Isolated from Clinical Urine Specimens}

The E. faecalis strains isolated in this study are listed in Table 1. In this study we used 11 strains, which showed various susceptibilities to faropenem, isolated from urine of different patients with UTIs seen at 5 hospitals in Kitakyushu, Japan between 1999 and 2004. Among the strains, E. faecalis Aef7 was isolated from the urine of an outpatient, but the others were isolated from urine of inpatients. We identified all the isolates used in this study as E. faecalis using the Vitek system (bioMerieux, France). The vancomycin resistance genes, vanA and $\operatorname{van} B$, were detected by PCR using specific primers [24, 25].

\section{Antimicrobial Agents and MIC Determination}

Antimicrobial agents were obtained as follows: faropenem, Daiichi Suntory Pharma Co., Ltd. Tokyo Japan; ampicillin, Nacalai Tesque, Inc. Kyoto Japan; imipenem, Banyu Tokyo Japan. We determined MICs on Mueller-Hinton agar (Difco) by a serial agar dilution method [26, 27].

\section{Beta-lactamase Activity}

Beta-lactamase activities of all the E. faecalis strains used in this study were examined following the method of O'Callaghan et al. with some modifications [28]. For hydrolysis studies, E. faecalis isolates were grown overnight at $37^{\circ} \mathrm{C}$ in BHI broth (Eiken Chemical Co., Ltd., Tokyo Japan) harvested and washed once in $200 \mathrm{ml}$ of $50 \mathrm{mM}$ sodium phosphate buffer ( $\mathrm{pH} 7.0$ ), and resuspended them in $20 \mathrm{ml}$ of sodium phosphate buffer. The suspension was sonically disrupted using an Ultrasonic disruptor UD201 (TOMY, Tokyo, Japan) and debris was removed by centrifugation. We tested the supernatant for beta-lactamase activity with nitrocefin (Oxoid, Ltd., United Kingdom). We used Escherichia coli ATCC 35218 which was a positive control strain for beta-lactamase activity [15].

\section{Isolation of Cytoplasmic Membranes}

Bacterial cytoplasmic membranes were prepared following the method of Spratt et al. [29]. In brief, cells were grown in $\mathrm{BHI}$ broth at $37^{\circ} \mathrm{C}$ overnight, collected in late exponential growth phase, and disrupted by sonication on ice. Residual cells were removed by centrifugation; protein concentration in the remaining supernatant was estimated using Lowry's method [30]. Then, membranes were stored at $-80^{\circ} \mathrm{C}$ in $50 \mathrm{mM}$ sodium phosphate, $\mathrm{pH} 7.0$, containing $10 \mathrm{mM} \mathrm{MgCl}{ }_{2}$.

\section{PBP Profiles}

We used Bocillin $\mathrm{FL}^{\circledR}$ (Molecular Probes, Inc., Eugene, Oregon), a commercially available fluorescent penicillin, to detect PBPs in bacterial membrane preparations following the method of Ono et al. [23, 31]. In brief, $20 \mu \mathrm{l}$ of a $20 \mathrm{mg} / \mathrm{ml} \mathrm{membrane} \mathrm{protein} \mathrm{suspension} \mathrm{was} \mathrm{incubated}$ with $15 \mu \mathrm{l}$ of $50 \mu \mathrm{M}$ Bocillin FL for 10 minutes at $30^{\circ} \mathrm{C}$. Proteins were then solubilized with sarcosyl, $180 \mathrm{mg} / \mathrm{ml}$ penicillin $\mathrm{G}$ was added, and the membrane fraction was obtained by centrifugation. Labeled membrane proteins were resolved by gel electrophoresis and visualized with UV light (ATTO AB1500, wavelength $312 \mathrm{~nm}$ ).

\section{Affinities of Antimicrobial Agents for PBPs}

We also used a competition assay to analyze the PBPs of these isolates. Membrane proteins $(20 \mathrm{mg} / \mathrm{ml})$ were incubated with increasing concentration of faropenem, ampicillin or imipenem (from 0.0625 to $32 \mu \mathrm{l} / \mathrm{ml}$ ) for 10 minutes at $30^{\circ} \mathrm{C}$. Samples were then incubated with $50 \mu \mathrm{M}$ Bocillin FL for another 10 minutes at $30^{\circ} \mathrm{C}$ and analyzed as described above. The $50 \%$ inhibitory concentration $\left(\mathrm{IC}_{50}\right)$ was taken to be the minimum concentration of unlabeled antibiotic which reduced binding of the Bocillin FL to a PBP by more than $50 \%$.

\section{Sequencing}

The entire pbp 4 genes of the E. faecalis strains were amplified by PCR using specific primers [23]. The PCR products were directly sequenced by the dideoxy chain termination method with an ABI PRISM 3130 DNA sequencer (PE Biosystems).

\section{Analysis of Genotypes by Pulsed-field Gel Electrophoresis (PFGE)}

All the genotypes of E. faecalis strains used in this study were analyzed by pulsed-field gel electrophoresis (PFGE) following the method of Kaufmann et al. [32] with some 





modifications. Briefly, the colonies of an overnight culture, grown at $37^{\circ} \mathrm{C}$ on blood agar, were mixed and suspended in $100 \mu 1$ of $50 \mathrm{mM}$ EDTA (pH 8.0). This suspension was mixed with $100 \mu \mathrm{l}$ of $1.2 \%$ agarose (chromosomal grade agarose, Bio-Rad Laboratories, USA) and pipetted into small plug molds. The cells suspended in the agarose plugs were lysed by incubation for 16 hours at $37^{\circ} \mathrm{C}$ in $0.6 \mathrm{ml}$ of $6.0 \mathrm{mM}$ Tris (pH 8.0)-0.1 M EDTA (pH 8.0) buffer containing $0.5 \%$ sarcosyl, $0.5 \%$ Brij $58,0.2 \%$ deoxycholate$\mathrm{Na}, 0.8 \mathrm{mg} / \mathrm{ml}$ of lysozyme (Wako Pure Chemical Industries, Ltd., Japan), $0.02 \mathrm{mg} / \mathrm{ml}$ of lysostaphin (Wako Pure Chemical Industries, Ltd., Japan) and $0.01 \mathrm{mg} / \mathrm{ml}$ of ribonuclease (Wako Pure Chemical Industries, Ltd., Japan). The lysis solution was replaced by a $0.5 \mathrm{ml}$ of $0.5 \mathrm{M}$ EDTA containing $1.0 \%$ sarcosyl and $0.25 \mathrm{mg} / \mathrm{ml}$ of proteinase $\mathrm{K}$ (Wako Pure Chemical Industries, Ltd., Japan), which was then incubated at $50^{\circ} \mathrm{C}$ for more than 16 hours. After being washed with $50 \mathrm{mM}$ EDTA, the plugs were incubated in $50 \mathrm{mM}$ EDTA containing $1.0 \mathrm{mM}$ phenylmethylsulfonyl fluoride (PMSF) at room temperature for 10 minutes. Then the DNA in the plugs was washed twice with $1.0 \mathrm{ml}$ of $50 \mathrm{mM}$ EDTA. The plugs were digested with SmaI (Nippon Gene Co., Japan), and products were separated on a $1.0 \%$ agarose gel (pulsed-field certified; Bio-Rad, Hercules, Calif.) by a CHEF Mapper pulsed-field gel electrophoresis system (Nippon Bio-Rad Laboratories, Japan). The electrophoresis conditions were as follows: pulse time ramped linearly from 3 to 45 seconds, run time was 22 hours, temperature was $14^{\circ} \mathrm{C}$, and constant voltage was $6 \mathrm{~V} / \mathrm{cm}$. PFGE results were interpreted in accordance with the criteria proposed by Tenover et al. [33].

\section{Results}

\section{Antimicrobial Susceptibility}

Table 1 shows the susceptibility of E. faecalis strains used in this study to faropenem, ampicillin, imipenem, vancomycin, linezolid, levofloxacin and sulfamethoxazole/ trimethoprim. MICs of faropenem against SEF96, ATCC29212, SVR250 and SVR251 were 0.5 to $1.0 \mu \mathrm{g} / \mathrm{ml}$. These 4 strains were defined as faropenem-susceptible strains in accordance with the faropenem susceptibility breakpoint, $2.0 \mu \mathrm{g} / \mathrm{ml}[2,12]$. MICs of faropenem against Aef7, Aef8, SVR9, SVR34 and SVR1080 were 4.0 to $8.0 \mu \mathrm{g} / \mathrm{ml}$ and these 5 strains were defined as faropeneminsensitive strains. MICs of faropenem against SVR1084, SVR1110 and SVR1119 were $32 \mu \mathrm{g} / \mathrm{ml}$ and these 3 strains were defined as faropenem-resistant strains. Faropenemsusceptible strains were also susceptible to ampicillin and imipenem (MICs of ampicillin, 1.0 to $2.0 \mu \mathrm{g} / \mathrm{ml}$; MICs of imipenem, $1.0 \mu \mathrm{g} / \mathrm{ml}$ ), faropenem-insensitive strains were also insensitive to ampicillin and imipenem (MICs of ampicillin, 4.0 to $8.0 \mu \mathrm{g} / \mathrm{ml}$; MICs of imipenem, 4.0 to $8.0 \mu \mathrm{g} / \mathrm{ml}$ ), and faropenem-resistant strains were also resistant to ampicillin and imipenem (MICs of ampicillin, $16 \mu \mathrm{g} / \mathrm{ml}$; MICs of imipenem, $32 \mu \mathrm{g} / \mathrm{ml}$ ) in accordance with the ampicillin susceptibility breakpoint, $8.0 \mu \mathrm{g} / \mathrm{ml}$ and the imipenem susceptibility breakpoint, $4.0 \mu \mathrm{g} / \mathrm{ml}[2,12]$. All the isolates were resistant to levofloxacin (MICs of levofloxacin, 16 to $512 \mu \mathrm{g} / \mathrm{ml}$ ) except the type strain ATCC29212 and the clinical isolate SEF96 (MICs of levofloxacin, $1.0 \mu \mathrm{g} / \mathrm{ml}$ ). MICs of vancomycin against the strains which had vanA (SVR9, SVR250, SVR251, SVR1080, SVR1084, SVR1110 and SVR1119) were more than $512 \mu \mathrm{g} / \mathrm{ml}$, and the MIC of vancomycin against $E$. faecalis SVR34 which had vanB was $64 \mu \mathrm{g} / \mathrm{ml}$. On the other hand, MICs of vancomycin against the strains having neither vanA nor vanB (ATCC29212, SEF96, Aef7 and Aef8) were 1.0 to $4.0 \mu \mathrm{g} / \mathrm{ml}$. MICs of sulfamethoxazole/ trimethoprim (19/1) against all the isolates except SVR34 (the MIC of sulfamethoxazole/trimethoprim, $19 / 1.0 \mu \mathrm{g} / \mathrm{ml}$ ) were $0.57 / 0.03$ to $2.47 / 0.13 \mu \mathrm{g} / \mathrm{ml}$. MICs of linezolid against all the isolates were 1.0 to $2.0 \mu \mathrm{g} / \mathrm{ml}$.

\section{Beta-lactamase Activity}

E. faecalis strains SVR9, Aef7, Aef8, SVR1080 and SVR1084 did not hydrolyze nitrocefin. In addition, it has already been reported that $E$. faecalis ATCC29212, SEF96, SVR250, SVR251, SVR34, SVR1110 and SVR1119 failed to hydrolyze nitrocefin [23].

\section{PBP Profiles}

We used a fluorescent penicillin (Bocillin FL) to detect PBP in membrane fractions prepared from all the $E$. faecalis isolates (the number of experimental trials, $\mathrm{n}=3$ ). PBPs of the isolates were expressed as the same five bands that corresponded to proteins of approximately $107 \mathrm{kDa}$, $81 \mathrm{kDa}, 76 \mathrm{kDa}, 72 \mathrm{kDa}$, and $67 \mathrm{kDa}$. Overproduction of PBPs was not seen previously in SVR34, SVR1110 and SVR1119 [23]. Likewise for the 5 new strains (SVR9, Aef7, Aef8, SVR1080 and SVR1084), we found no evidence of overproduction of any PBP.

\section{Affinities of the Antibiotics for PBPs}

We performed competition assays to measure the affinity of faropenem, ampicillin and imipenem for each PBP $(n=3)$. $\mathrm{IC}_{50}(\mu \mathrm{g} / \mathrm{ml})$ is expressed as the minimum concentration of antibiotics required to reduce Bocillin FL binding to the PBP by more than $50 \%$. There were no differences in $\mathrm{IC}_{50} \mathrm{~s}$ of faropenem against any PBPs of all strains except for PBP4. There were obvious differences in PBP4 saturation 
Table 2 Inhibition of binding Bocillin FL to PBPs by antimicrobial agents used in this study

\begin{tabular}{|c|c|c|c|c|c|c|}
\hline \multirow{2}{*}{ Drug } & \multirow{2}{*}{ E. faecalis strain } & \multicolumn{4}{|c|}{$\mathrm{IC}_{50}(\mu \mathrm{g} / \mathrm{ml})$} & \multirow{2}{*}{$\operatorname{MIC}(\mu \mathrm{g} / \mathrm{ml})$} \\
\hline & & PBP1 & PBP3 & PBP4 & PBP5 & \\
\hline \multirow[t]{12}{*}{ FRPM } & ATCC29212 & 8 & 0.5 & 1 & $>32$ & 1 \\
\hline & SEF96 & 16 & 0.5 & 1 & $>32$ & 1 \\
\hline & SVR250 & 8 & 1 & 1 & $>16$ & 0.5 \\
\hline & SVR251 & 4 & 0.5 & 0.5 & $>32$ & 0.5 \\
\hline & SVR9 & 8 & 1 & 2 & $>32$ & 4 \\
\hline & Aef7 & 8 & 1 & 2 & $>32$ & 4 \\
\hline & Aef8 & 8 & 0.5 & 2 & $>32$ & 8 \\
\hline & SVR34 & 8 & 1 & 2 & $>32$ & 8 \\
\hline & SVR1080 & 8 & 0.5 & 2 & $>32$ & 8 \\
\hline & SVR1084 & 4 & 0.5 & 32 & $>32$ & 32 \\
\hline & SVR1110 & 8 & 1 & 32 & $>32$ & 32 \\
\hline & SVR1119 & 8 & 0.5 & 16 & $>32$ & 32 \\
\hline \multirow[t]{12}{*}{ AMP } & ATCC29212 & 1 & 0.5 & 0.25 & $>16$ & 1 \\
\hline & SEF96 & 1 & 0.5 & 0.25 & $>16$ & 2 \\
\hline & SVR250 & 2 & 0.5 & 1 & $>8$ & 1 \\
\hline & SVR251 & 2 & 0.5 & 0.25 & $>8$ & 2 \\
\hline & SVR9 & 2 & 0.5 & 4 & $>32$ & 4 \\
\hline & Aef7 & 2 & 0.5 & 4 & $>32$ & 4 \\
\hline & Aef8 & 2 & 0.5 & 4 & $>32$ & 4 \\
\hline & SVR34 & 2 & 0.5 & 4 & $>32$ & 4 \\
\hline & SVR1080 & 2 & 0.5 & 4 & $>32$ & 8 \\
\hline & SVR1084 & 1 & 1 & 8 & $>32$ & 16 \\
\hline & SVR1110 & 1 & 0.5 & 8 & $>32$ & 16 \\
\hline & SVR1119 & 1 & 0.5 & 8 & $>32$ & 16 \\
\hline \multirow[t]{12}{*}{ IPM } & ATCC29212 & 0.5 & 0.25 & 0.125 & $>16$ & 1 \\
\hline & SEF96 & 0.5 & 0.25 & 0.25 & $>16$ & 1 \\
\hline & SVR250 & 1 & 0.5 & 0.25 & $>32$ & 1 \\
\hline & SVR251 & 0.5 & 0.25 & 0.5 & $>32$ & 1 \\
\hline & SVR9 & 0.5 & 0.5 & 4 & $>32$ & 4 \\
\hline & Aef7 & 1 & 0.5 & 4 & $>32$ & 4 \\
\hline & Aef8 & 1 & 0.25 & 4 & $>32$ & 4 \\
\hline & SVR34 & 0.5 & 0.25 & 4 & $>32$ & 8 \\
\hline & SVR1080 & 0.5 & 0.25 & 4 & $>32$ & 8 \\
\hline & SVR1084 & 0.5 & 0.5 & 16 & $>32$ & 32 \\
\hline & SVR1110 & 1 & 0.5 & 32 & $>32$ & 32 \\
\hline & SVR1119 & 1 & 0.25 & 16 & $>32$ & 32 \\
\hline
\end{tabular}

Abbreviations: FRPM, faropenem; AMP, ampicillin; IPM, imipenem.

$I C_{50}$, Minimum concentration of unlabeled antibiotic which reduces binding of Bocillin FL to a PBP by more than $50 \%$.

for faropenem between faropenem-susceptible strains and faropenem-resistant strains. MICs and $\mathrm{IC}_{50} \mathrm{~s}$ of faropenem against PBP4 of faropenem-resistant strains were obviously higher than those of faropenem-susceptible strains. Similar to MICs and $\mathrm{IC}_{50} \mathrm{~s}$ of faropenem, MICs and $\mathrm{IC}_{50} \mathrm{~s}$ of ampicillin or imipenem against PBP4 of faropenemresistant strains were obviously higher than those of faropenem-susceptible strains (Tables 2, 3).

The $\mathrm{IC}_{50} \mathrm{~s}$ of faropenem, ampicillin and imipenem against PBP4 of all strains had a positive correlation with 
Table 3 Substitutions in amino acid sequences of pbp4 of E. faecalis strains used in this study compared with E. faecalis SEF96, MICs and $\mathrm{IC}_{50}$ s of three antimicrobial agents for the strains

\begin{tabular}{|c|c|c|c|c|c|c|c|}
\hline \multirow{2}{*}{ E. faecalis strain } & \multicolumn{5}{|c|}{ Alteration at position } & \multirow{2}{*}{$\begin{array}{l}\text { MICs }(\mu \mathrm{g} / \mathrm{ml}) \text { of } \\
\text { FRPM/AMP/IPM }\end{array}$} & \multirow{2}{*}{$\begin{array}{c}\qquad \mathrm{C}_{50} \mathrm{~s}(\mu \mathrm{g} / \mathrm{ml}) \text { of } \\
\text { FRPM/AMP/IPM to PBP4 }\end{array}$} \\
\hline & 50 & 369 & 475 & 520 & 605 & & \\
\hline SEF96 & Thr & Val & Leu & Pro & Tyr & $1 / 2 / 1$ & $1 / 0.25 / 0.25$ \\
\hline ATCC29212 & & Ala & & & & $1 / 1 / 1$ & $1 / 0.25 / 0.125$ \\
\hline SVR250 & Ile & & & & & $0.5 / 1 / 1$ & $1 / 1 / 0.25$ \\
\hline SVR251 & Ile & & & & & $0.5 / 2 / 1$ & $0.5 / 0.25 / 0.5$ \\
\hline SVR9 & & & & Ser & & $4 / 4 / 4$ & $2 / 4 / 4$ \\
\hline Aef7 & & & Met & & & $4 / 4 / 4$ & $2 / 4 / 4$ \\
\hline Aef8 & & & Met & & & $8 / 4 / 4$ & $2 / 4 / 4$ \\
\hline SVR34 & & & & Ser & & $8 / 4 / 8$ & $2 / 4 / 4$ \\
\hline SVR1080 & & & & & His & $8 / 8 / 8$ & $2 / 4 / 4$ \\
\hline SVR1084 & & & & Ser & His & $32 / 16 / 32$ & $32 / 8 / 16$ \\
\hline SVR1110 & & & & Ser & His & $32 / 16 / 32$ & $32 / 8 / 32$ \\
\hline SVR1119 & & & & Ser & His & $32 / 16 / 32$ & $16 / 8 / 16$ \\
\hline
\end{tabular}

Abbreviations: FRPM, faropenem; AMP, ampicillin; IPM, imipenem.

MICs of the agents, respectively.

\section{Deduced Amino Acid Sequences of PBP4 Based on Sequences of $p b p 4$ Genes}

We amplified the pbp 4 genes of the E. faecalis isolates and sequenced the entire $p b p 4$ genes corresponding to amino acids 1 to 680 , to identify mutations that may account for the differences in the affinity of this protein for antibiotics among the faropenem-susceptible, -insensitive and -resistant strains. SVR1084, a faropenem-resistant strain (the MIC of faropenem, $32 \mu \mathrm{g} / \mathrm{ml}$, the $\mathrm{IC}_{50}$ of faropenem against PBP4, $32 \mu \mathrm{g} / \mathrm{ml}$ ) had the same two amino acid substitutions (Pro520Ser and Tyr605His) in the pbp4 as faropenem-resistant strains SVR1110 and SVR1119, the sequences of which had been previously reported, compared to the amino acid sequences of SEF96, a penicillin-susceptible strain enrolled in GenBank [23]. Four faropenem-insensitive strains had one out of three single amino acid substitutions in PBP4, which were Leu475Met, Pro520Ser or Tyr605His (Table 3).

\section{Analysis of Genotypes by Pulsed-field Gel Electrophoresis (PFGE)}

In accordance with the criteria proposed by Tenover et al. [33], we did not find any indistinguishable genotypes by PFGE, but SVR1080, SVR1084, SVR1110 and SVR1119 appeared to have possibly related genotypes $(n=3)$ (Fig. 1).

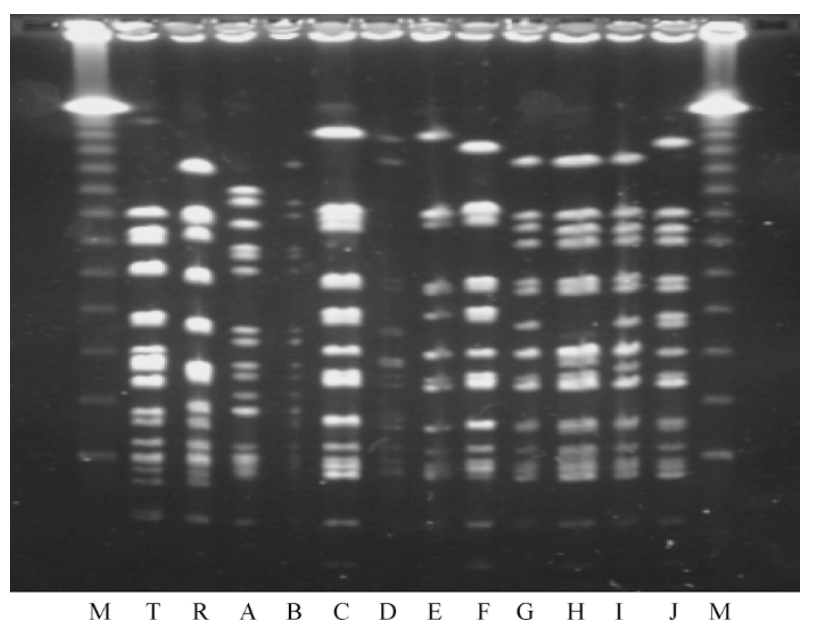

Fig. 1 Pulsed-field gel electrophoresis (PFGE) typing.

Total genetic DNA was digested with Sma I and subjected to PFGE. Lane M, DNA marker; Lane T, type strain ATCC29212; Lane $R$, reference strain SEF96; Lane A, SEF250; Lane B, SEF251; Lane C, SVR9; Lane D, Aef7; Lane E, Aef8; Lane F, SVR34; Lane G, SVR1080; Lane H, SVR1084; Lane I, SVR1110; Lane J, SVR1119.

\section{Discussion}

Faropenem is a member of the penems which form a discrete class of beta-lactams. Faropenem has potent antimicrobial activity against most of the causative isolates 
of UTIs, especially against $E$. faecalis which is intrinsically resistant to cephems [3,4]. E. faecalis is susceptible to ampicillin and is also susceptible to imipenem and faropenem, a difference from E. faecium. In fact, most of the E. faecalis isolates are treatable with these antibiotics $[1,2,14]$. We have found few reports of faropenemresistant $E$. faecalis, but we isolated faropenem-resistant E. faecalis strains in the urine of patients with UTIs. Here, we studied the mechanisms of resistance to faropenem in these isolates.

Three mechanisms of penicillin resistance have been reported in E. faecalis. Beta-lactamase production was first reported in 1983 [15], and there have been several subsequent reports of beta-lactamase-producing penicillin resistance in E. faecalis [16 19]; nevertheless, betalactamase production is a very rare mechanism in enterococci [3, 4, 34]. E. faecalis strains carrying blaR1 and blal, staphylococcal beta-lactamase regulatory genes, produced small amounts of class A beta-lactamases [17, 35]; these enzymes do not hydrolyze either carbapenems or penems $[2,4,5]$. Thus, even beta-lactamase-producing E. faecalis are expected to be susceptible to faropenem and imipenem.

Another mechanism of resistance is overproduction of PBP4 or PBP5 [20 22]. E. faecalis JH2-2r (the MIC of benzylpenicillin, $75 \mu \mathrm{g} / \mathrm{ml}$ ) was selected from E. faecalis JH2-2 by successive growth on BHI plates containing increasing concentrations of benzylpenicillin. JH2-2r showed overproduction of PBP4 compared with JH2-2. In their study, however, the cloning and sequencing of $p s r$-like gene, which was a repressor-encoding gene (for PBP5 synthesis repressor), from both $\mathrm{Jh} 2-2$ and $\mathrm{JH} 2-2 \mathrm{r}$ indicated that they were identical [20]. Clinical isolates of ampicillinresistant E. faecalis (the MIC of ampicillin, $32 \mu \mathrm{g} / \mathrm{ml}$ ), which had no beta-lactamases, showed overproduction of PBP5 [21]. MICs for ampicillin in mutant derivatives of E. faecalis 56 carrying a plasmid coding for $p b p 5$ were $64 \mu \mathrm{g} / \mathrm{ml}$, which were 16 times higher than that of parent strain E. faecalis 56 (the MIC of ampicillin, $4.0 \mu \mathrm{g} / \mathrm{ml}$ ). The mutant derivatives also showed overproduction of PBP5 compared with the parent strain [22].

The other mechanism of resistance is decreasing affinities of penicillins for PBP4 by the occurrence of point mutations in the penicillin-recognition domain [23]. E. faecalis PBP4 is a membrane protein composed of 680 amino acids with three distinct modules. One is a penicillin-binding module (amino acids 350 to 680) located towards the $C$-terminus, in which three typical penicillinbinding motifs (the active-site-defining motifs), STFK, SDN and KTG, were identified as the main target of betalactams. The others are a non-penicillin-binding module (amino acids 40 to 349 ) and an uncleaved $N$-terminal segment that acts as a membrane-spanning (anchoring) domain (amino acids 1 to 39) [22, 23]. Ono et al. reported the mechanisms of resistance to ampicillin and imipenem in vancomycin-resistant $E$. faecalis isolated from clinical specimens [23]. These strains lacked beta-lactamases and did not overproduce PBPs. It was also reported that the MICs of ampicillin and imipenem for E. faecalis SVR1119 and E. faecalis SVR1119S, which was cured of a vanAcarrying plasmid from SVR1119, were identical, suggesting that vanA was not involved in the development of resistance to ampicillin and imipenem in the strains.

The MICs of ampicillin or imipenem for ampicillin, imipenem-insensitive strains increased twice or 8 times (ampicillin) and 16 times (imipenem) compared with those for ampicillin, imipenem-susceptible strains (MICs of ampicillin, $1.0 \mu \mathrm{g} / \mathrm{ml}$; MICs of imipenem, 0.5 to $1.0 \mu \mathrm{g} / \mathrm{ml}$ ), respectively. The MICs of ampicillin or imipenem for ampicillin, imipenem-resistant strains increased 32 to 64 times compared with those for ampicillin, imipenemsusceptible strains. On the other hand, the $\mathrm{IC}_{50} \mathrm{~s}$ of ampicillin or imipenem against PBP4 for ampicillin, imipenem-insensitive strains also increased twice or 4 to 8 times compared with those for ampicillin, imipenemsusceptible strains, respectively. The $\mathrm{IC}_{50} \mathrm{~s}$ of ampicillin or imipenem against PBP4 for ampicillin, imipenemresistant strains also increased 8 to 32 times or 32 to 256 times compared with those for ampicillin, imipenemsusceptible strains, respectively. Sequencing of $p b p 4$ for the strains showed that Pro-520 was changed to a Ser residue in ampicillin, imipenem-insensitive strains (MICs of ampicillin, $8.0 \mu \mathrm{g} / \mathrm{ml}$; MICs of imipenem, $4.0 \mu \mathrm{g} / \mathrm{ml})$, and Pro-520 was replaced with a Ser residue. The same Pro520Ser alteration was seen at position 520 and Tyr-605 was replaced with a His residue in ampicillin, imipenemresistant strains (MICs of ampicillin, $16 \mu \mathrm{g} / \mathrm{ml}$; MICs of imipenem, $32 \mu \mathrm{g} / \mathrm{ml}$ ) [23].

In the present study, none of the isolates used produced any beta-lactamases and none overproduced PBPs. All faropenem-resistant strains had vanA; 3 faropeneminsensitive strains had van $A$ and vanB; 2 faropenemsusceptible strains had van $A$ and 2 faropenem-insensitive strains did not have either vanA or vanB. In addition, it was already reported that there were no differences between the susceptibilities of ampicillin and imipenem for SVR1119 and SVR1119S [23]. Therefore, vanA and vanB were not involved in development of resistance to faropenem, ampicillin and imipenem in these strains (Table 1).

In faropenem-insensitive strains, we found a single point mutation at positions 475,520 or 605 in the penicillin binding module; these amino acid substitutions produced 
similar effects on the MICs and $\mathrm{IC}_{50} \mathrm{~s}$ for faropenem. In the faropenem-resistant strains, we found point mutations at both position 520 and 605 in the penicillin binding module; this double substitution also produced similar effects on the MICs and $\mathrm{IC}_{50}$ s for faropenem against PBP4 (Tables 2, 3). These point mutations occurred in the region between the active-site-defining motifs, STFK, SDN and KTG, in the penicillin-binding module. SVR1080 had a novel single Tyr605His amino acid substitution and both Aef7 and Aef8 also had another novel single Leu475Met amino acid substitution; all three strains were faropenem-insensitive. Two other faropenem-insensitive strains SVR9 and SVR34 had the same single Pro520Ser amino acid substitution [23]. It has been reported that point mutations outside the penicillin-binding domain of PBP4 (E. faecalis ATCC29212, SVR250 and SVR251) had no effect on MICs and $\mathrm{IC}_{50} \mathrm{~S}$ of ampicillin and imipenem for these strains [23]. In the present study, point mutations outside the penicillinbinding domain of PBP4 also failed to alter MICs and $\mathrm{IC}_{50} \mathrm{~s}$ of faropenem (Table 3). Thus, it is considered that MICs and $\mathrm{IC}_{50} \mathrm{~s}$ of faropenem as well as ampicillin and imipenem against PBP4 were increased by point mutations that occurred in the penicillin binding module of PBP4.

SVR1080, faropenem-insensitive strain, is similar by PFGE to the resistant strain, SVR1110 (Fig. 1). In the insensitive strains, Pro-520 was replaced with Ser or Tyr-605 was replaced with His, but in the resistant strains both changes (Pro520Ser and Tyr605His) were observed in the region between the active-site-defining motifs, STFK, SDN and KTG. Therefore, it is suggested that an insensitive strain, SVR1080 was transformed to the more resistant strain, SVR1110 by acquiring an additional point mutations.

We conclude that development of resistance to faropenem as well as ampicillin and imipenem depends on decreasing affinities of the antimicrobial agents for PBP4 by the occurrence of one or two point mutations at position 475, 520 or 605 in the penicillin binding module. In this present study, we would like to stress here that one point mutation in the PBP4 of E. faecalis shows low resistance to the aforementioned antibiotics, but just two point mutations lead to drastically higher resistance. This study is the first report regarding the mechanisms of resistance to faropenem in E. faecalis. Faropenem plays an important role in the treatment of a wide range of infections, especially UTIs in Japan. If faropenem-resistant E. faecalis strains continue to increase, these strains will cause serious problems for treatment of the enterococcal infections. Strict control and prevention of nosocomial infections should be required.

Acknowledgments In carrying out this research, we received
E. faecalis strains used in this study from the members of HIBIKI Research Group for Clinical Microbiology (HRGCM), to whom we would like to express our sincere thanks. Especially, we thank Tomoko Kobayashi of Testing Department, KYURIN CORPORATION, Japan, for her helpful advice and contribution to this research, Dr. Haruhisa Saku of Aishinkai Kokuraitoudu Hospital, Japan, for his helpful suggestions, Kaoru Shiozaki and Shizuka Lee of Department of Urology, School of Medicine, University of Occupational and Environmental Health, Japan, for their kind technical support, and Dr. Shunichi Kajioka of Department of Urology, Graduate School of Medical Sciences, Kyushu University, Japan, for his helpful advice and suggestions.

\section{References}

1. Jeremy M, Hamilton-Miller T. Chemical and microbiologic aspects of penems, a distinct class of beta-lactams: focus on faropenem. Pharmacotherapy 23: 1497-1507 (2003)

2. Muratani T, Matsumoto T. Bacterial resistance to antimicrobials in urinary isolates. Int $\mathrm{J}$ Antimicrob Agents 24S: S28-S31 (2004)

3. Cetinkaya Y, Falk P, Mayhall CG. Vancomycin-resistant enterococci. Clin Microbiol Rev 13: 686-707 (2000)

4. Poole K. Resistance to beta-lactam antibiotics. Cell Mol Life Sci 61: 2200-2223 (2004)

5. Muratani $\mathrm{T}$, Iihara $\mathrm{K}$, Matsumoto $\mathrm{T}$, et al. Faropenem $300 \mathrm{mg} 3$ times daily versus levofloxacin $100 \mathrm{mg} 3$ times daily in the treatment of urinary tract infections in patients with neurogenic bladder and/or benign prostatic hypertrophy. J Jpn Assoc Infect Dis 76: 928-938 (2002)

6. Koch S, Hufnagel M, Huebner J, et al. Enterococcal infections: host response, therapeutic, and prophylactic possibilities. Vaccine 22: 822-830 (2004)

7. Karina M, Butler MB. Enterococcal infection in children. Semin Pediatr Infect Dis 17: 128-139 (2006)

8. Morrison AJ, Wenzel RP. Nosocomial urinary tract infections due to Enterococcus. Ten years' experience at a university hospital. Arch Intern Med 146: 1549-1551 (1986)

9. Takahashi S, Hirose T, Tsukamoto T, et al. Analysis of cross infection using genomic fingerprinting in nosocomial urinary tract infection caused by Enterococcus faecalis. J Infect Chemother 5: 46-48 (1999)

10. Langley JM, Hanakowski M, Leblanc JC. Unique epidemiology of nosocomial urinary tract infection in children. Am J Infect Control 29: 94-98 (2001)

11. Bitsori M, Maraki S, Galanakis E, et al. Communityacquired enterococcal urinary tract infections. Pediatr Nephrol 20: 1583-1586 (2005)

12. Centers for Disease Control and Prevention. Nosocomial enterococci resistant to vancomycin-United States, 19891993. J Am Med Assoc 270: 1796 (1993)

13. Burke JP. Hospitals enter the war against antibiotic resistance. Curr Opin Infect Dis 8: 269-271 (1995)

14. Woodcock JM, Andrews JM, Wise R, et al. The in-vitro 
activity of faropenem, a novel oral penem. J Antimicrob Chemother 39: 35-43 (1997)

15. Murray BE, Mederski-Samoraj B. A new mechanism for in vitro penicillin resistance in Streptococcus faecalis. J Clin Investig 72: 1168-1171 (1983)

16. Handwerger S, Perlman DC, McAuliffe V. Concomitant high-level vancomycin and penicillin resistance in clinical isolates of enterococci. Clin Infect Dis 14: 655-661 (1992)

17. Okamoto R, Okubo $T$, Inoue $M$. Detection of genes regulating beta-lactamase production in Enterococcus faecalis and Staphylococcus aureus. Antimicrob Agents Chemother 40: 2550-2554 (1996)

18. Murray BE, Church DA, Mederski-Samoraj B, et al. Comparison of two beta-lactamase producing strains of Streptococcus faecalis. Antimicrob Agents Chemother 30: 861-864 (1986)

19. Murray BE, Singh KV, Goldstein FW, et al. Evidence for clonal spread of a single strain of beta-lactamase-producing Enterococcus faecalis to six hospitals in five states. J Infect Dis 163: 780-785 (1991)

20. Duez C, Zorzi W, Coyette J, et al. The penicillin resistance of Enterococcus faecalis JH2-2r results from an overproduction of the low-affinity penicillin-binding protein PBP4 and does not involve a psr-like gene. Microbiology 147: 2561-2569 (2001)

21. Cercenado E, Vicente MF, Sanchez-Rubiales M, et al. Characterization of clinical isolates of beta-lactamasenegative, highly ampicillin-resistant Enterococcus faecalis. Antimicrob Agents Chemother 40: 2420-2422 (1996)

22. Signoretto C, Canepari P. Paradoxical effect of inserting, in Enterococcus faecalis penicillin-binding protein 5, an amino acid box responsible for low affinity for penicillin in Enterococcus faecium. Arch Microbiol 173: 213-219 (2000)

23. Ono S, Muratani T, Matsumoto T. Mechanisms of resistance to imipenem and ampicillin in Enterococcus faecalis. Antimicrob Agents Chemother 49: 2954-2958 (2005)

24. Elsayed S, Hamilton N, Mulvey M, et al. Improved primer design for multiplex PCR analysis of vancomycin-resistant Enterococcus spp. J Clin Microbiol 39: 2367-2368 (2001)
25. Kariyama R, Mitsuhata R, Kumon H, et al. Simple and reliable multiplex PCR assay for surveillance isolates of vancomycin-resistant enterococci. J Clin Microbiol 38: 3092-3095 (2000)

26. National Committee for Clinical Laboratory Standards. Methods for dilution antimicrobial susceptibility tests for bacteria that grow aerobically. Approved standard M7-A3. National Committee for Clinical Laboratory Standards, Villanova, Pa. (1993)

27. National Committee for Clinical Laboratory Standards. Methods for dilution antimicrobial susceptibility tests for bacteria that grow aerobically, 5th ed. Approved standard M7-A5. National Committee for Clinical Laboratory Standards, Wayne, Pa. (2002)

28. O'Callaghan $\mathrm{CH}$, Morris A, Shingler AH, et al. Novel method for detection of beta-lactamases by using a chromogenic cephalosporin substrate. Antimicrob Agents Chemother 1: 283-288 (1972)

29. Spratt B, Pardee AB. Penicillin-binding proteins and cell shape in Escherichia coli. Nature 254: 516-517 (1975)

30. Lowry $\mathrm{OH}$, Rosebrough $\mathrm{NJ}$, Randall RJ, et al. Protein measurement with the Folin phenol reagent. J Biol Chem 193: 265-275 (1951)

31. Zhao G, Meier TI, Blaszczak LC, et al. Bocillin FL, a sensitive and commercially available reagent for detection of penicillin-binding proteins. Antimicrob Agents Chemother 43: 1124-1128 (1999)

32. Kaufmann ME. Pulsed-field gel electrophoresis. Methods Mol Med 15: 17-31 (1998)

33. Tenover FC, Arbeit RD, Goering RV, et al. Interpreting chromosomal DNA restriction patterns produced by pulsedfield gel electrophoresis: criteria for bacterial strain typing. J Clin Microbiol 33: 2233-2239 (1995)

34. Signoretto C, Boaretti M, Canepari P. Peptidoglycan synthesis by Enterococcus faecalis penicillin binding protein 5. Arch Microbiol 170: 185-190 (1998)

35. Murray BE. Beta-lactamase-producing enterococci. Antimicrob Agents Chemother 36: 2355-2359 (1992) 